\title{
An Ethnobotanical Study of Medicinal Plants used against Jaundice by Tea Tribes of Morigaon District, Assam (India)
}

\author{
Rakhi Bhattacharyya ${ }^{1 *}$, Krishna Kanta Medhi ${ }^{1}$, Sashin Kumar Borthakur ${ }^{2}$ \\ and Sarat Borkataki ${ }^{1}$ \\ 1'Department of Botany, Nowgong College, Nagaon - 782001, Assam, India; \\ rakhibhattacharyya06@gmail.com \\ 2Department of Botany, Gauhati University, Guwahati - 781014, Assam, India
}

\begin{abstract}
The present study was conducted to document the ethnomedicinal plants used against jaundice by the tea tribes of Morigaon district of Assam, India. An ethnomedicinal field study was carried out from June 2016 - July 2017. Information was gathered by using a semi-structured questionnaire about the traditional knowledge of medicinal plants used against jaundice by the tea tribes of Morigaon district of Assam. Documented data was evaluated using the quantitative ethno-botanical indices of fidelity level (FL), Use Value (UV) and Family Use Value (FUV). From the ethno-botanical investigation, a total of 39 species of plants covering 36 genera and 27 families respectively have been enumerated. A total of 53 informants aged from 20-75 years were interviewed to record the ethnomedicinal data. Lamiaceae was the dominant family. Among the plant portions, leaves were most frequently used. Among the 39 medicinal plant species recognized mostly were herbs. The plants species having the highest use value were Drymaria cordata trailed by Xylosma longifolia and Achyranthes aspera, Aegle marmelos, Alstonia scholaris and Justicia gendarussa. The fidelity level was $100 \%$ for Achyranthes aspera, Cheilocostus speciosus, Clerodendrum infortunatum, Justicia gendarussa, Lawsonia inermis, Coffea benghalensis and Saccharum officinarum. The tea tribes of Morigaon district still relies on herbal therapies for curing jaundice. Coffea benghalensis has not been previously reported as a remedy of jaundice from Northeast India. Further research is needed to investigate the phytochemistry and pharmacological effectiveness of the plant species that could be the basis for the isolation and development of some novel phyto-therapeutic active compounds in the future.
\end{abstract}

Keywords: Ethnobotanical Study, Jaundice, Medicinal Plants, Morigaon District, Tea Tribes

\section{Abbreviations Used}

WHO: World Health Organization; FL: Fidelity Level; UV: Use Value; FUV: Family Use Value; Ass: Assamese name; TT: Tea Tribal name

\section{Introduction}

Ethno-medicine has evolved and developed since the prehistoric period. Traditional medicine is still the main source for treating health related problems around the planet 1 . Despite globalization and modernization, about $60-85 \%$ of the world's population in the developing countries relies on natural and traditional medicine ${ }^{2}$. According to WHO, around 21,000 plant species have been estimated which can be potentially used for medicinal purpose ${ }^{3,4}$. Ethnic groups store an immense knowledge on traditional herbal medicine and this need to be properly documented 5 . Ethnobotanical studies are the key sources for the discovery of novel drugs from

${ }^{*}$ Author for correspondence 
the plant species ${ }^{6}$. India is considered as the leading producer of medicinal plants and represents one of the greatest reservoirs of ethno-botanical wealth ${ }^{7} \underline{\underline{7}}$. Nearly, $65 \%$ of the Indian population depends on traditional therapies ${ }^{9}$. North-east India including Assam is considered as the paradise for anthropologists and ethno-botanists as it has a rich vegetation wealth due to topographic and climatic specificity and is among the most diverse regions in the world with more than 150 tribes existing and the tea tribes are one among them $\underline{10}, \underline{11}$.

Jaundice is a complex ailment caused due to the malfunctioning of the liver, which results in the excessive level of yellow-orange bile pigment i.e. bilirubin, which gets deposited in the tissues of skin, mucous membranes and the sclera ${ }^{12}$. The word jaundice is a derivative of French word 'Jaune' that means 'yellow'. The causes of jaundice are either acquired or congenital 13 . Jaundice is a symptom rather than a disease. There are several possible causes of jaundice viz., hepatitis (A, B, C, D and E), obstruction of bile ducts, liver cirrhosis, gall-bladder stones, inflammation of the liver, pancreatic cancer, alcoholic liver disease, tuberculosis, typhoid, malaria, haemolytic anaemia, yellow fever, certain medication, neonatal jaundice and pregnancy. A large number of plant species claim to possess the liver protecting activity $\underline{14}$. Almost 160 phyto-compounds from 101 plants claim to possess the hepatoprotective activity $\underline{15}$. The tea garden community of Assam uses a number of self-remedial medications and herbal therapies for the treatment of jaundice. This acquired knowledge about the traditional medicinal plants may be helpful for developing new medicines for Jaundice by identifying novel bioactive compounds ${ }^{16}$. The chief objective of the study was to measure and document the richness of indigenous knowledge on medicinal plants against jaundice used by the tea tribes of Morigaon district of Assam, so that the documented plant species can be used for phytochemical and pharmacological discoveries in the future.

\section{Materials and Method}

\subsection{Study Area and the People}

An explorative investigation was undertaken in the tea gardens of Morigaon district of Assam, which is located in the central region of Assam. The district lies between $26^{\circ} 45^{\prime}$ North Latitudes and $93^{\circ} 50^{\prime}$ East Longitude, covering a geographical area of 1,704 square kilometers (658 square miles). The Morigaon district is bordered by river Brahmaputra in the North, West Karbi-Anglong district in the south, Nagaon district in the east, and the Kamrup district in the west, where it also shares a small border with Meghalaya. The climate is of general monsoon type with an average rainfall of about $1,753 \mathrm{~mm}$. The tea tribes of Morigaon district were originally from Andhra Pradesh, Bihar, Jharkhand, Orissa, Tamilnadu and West Bengal including Bhumij, Bhil, Bhuyan, Kohar, Kurmi, Khariya, Garh, Munda, Mahanty, Tanti, Telenga, Lohar, Orang, Oriya, Sundi, Ghatwal, Goala, Kalindi, Kaul, Santhal, and Gonju tribes $\underline{17}, \underline{18}$. The main languages spoken by the tea tribes are Assamese, Baganiya bhasa / Tea tribal language (amalgamation of Bengali, Oriya and Shadri languages). The major sources of livelihood for the tea tribes are working as laborers in the tea gardens and also as traditional herbal practitioners (bej). The present communication provides results of indigenous uses of phyto-medicines against jaundice used by the tea tribes of the Morigaon district.

\subsection{Documentation and Collection of Ethnomedicinal Data}

Extensive field surveys conducted in the tea gardens of Morigaon district during June 2016 -September 2017 and maximum information provided by the tea tribes were collected (Figure 1). In total 53 informants were interviewed within the age group of 20-75 years. The

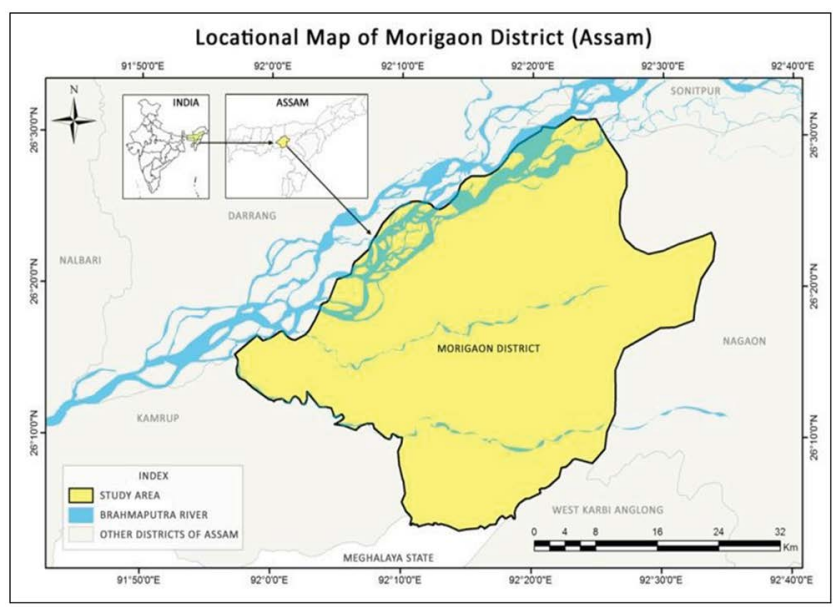

Figure 1. Geographical location of Morigaon district. 
information was obtained through semi-structured questionnaires, interviews, consultations and group discussions on the medicinal use of the plants against jaundice and detailed information about mode of preparation (i.e., decoction, infusion, juice or extract, paste, powder and external use) was documented $\underline{19}, \underline{20}$. Prior permission was sought from the concerned authorities of the tea gardens for the collection of the ethnomedicinal data along with the plants and also the related plant parts required from the study area. Before proceeding with the interview, consent of the respondents was also obtained to carry out the study. The collected specimens were processed and mounted in a herbarium using convenient techniques and were cross-checked and identified with the help of relevant floras and were matched at the Gauhati University Herbarium and Nowgong College Herbarium and then their identity was confirmed ${ }^{21}, \underline{22}$. Nomenclatures of plants were updated using The Plant List and the specimens are deposited at Department of Botany, Nowgong College (Nagaon) for future references $\underline{23}$. All the recorded plant species are presented in tabular format, alongside the family, common names and the ethnomedicinal usage information.

\subsection{Quantitative Analysis of Ethnomedicinal Data}

An illustrative method of using the frequencies and percentages was used to evaluate the socio-demographic data of the informants. The ethnomedicinal usage of plants was quantitatively accessed using the Fidelity Level (FL), Use Value (UV) and Family Use Value (FUV).

\subsubsection{Fidelity Level (FL)}

The Fidelity Level (FL) was calculated to determine the percentage of informants who mentioned the uses of certain plant species to treat a particular ailment in the study area.

$$
F L(\%)=\left(\frac{l p}{l u}\right) \times 100
$$

Where, lp is the number of informants who independently claimed the utilization of a plant species for the same major ailment and $\mathrm{lu}$ is the number of informants who mentioned the plant for any major ailment $\underline{24}, \underline{25}$.

\subsubsection{Use Value (UV)}

The Use Value (UV) determines the relative importance of plants known in locally. It was calculated using the following formula ${ }^{26}$.

$$
U V=\frac{\sum U i}{N}
$$

Where, $U_{i}$ is the number of uses mentioned by each informant for a given species and $\mathrm{N}$ is the total number of informants.

\subsubsection{Family Use Value (FUV)}

The significance of the plant families were identified by calculating the Family Use Value (FUV) using the following formula ${ }^{27}$.

$$
F U V=\frac{U V s}{N s}
$$

Where, UVs is the use value of the species and Ns is the total number of species within each family.

\section{Results and Discussion}

\subsection{Socio-Demographic Data of the Study}

A total of 53 informants have participated in the present study. The age groups of the informants were confined between 20 and 75 years are represented in Table 1.

A total of 53 informants were interviewed and all were indigenous people with ages ranging from 20 years to 75 years (Table 1). They comprised of $73.58 \%$ men $26.41 \%$ women. A large number of people were in the age of $50-60$ years $(33.96 \%)$ and $40-50$ years $(24.52 \%)$. Most of the people in the study area were laborers (79.24\%) and traditional health practitioners (13.2\%). Regarding the level of education, $67.92 \%$ were illiterate and $32.07 \%$ were literate.

\subsection{Plant Species, Botanical Families, Mode of Remedy Preparation and Quantitative Values}

The plant species recorded in the present study are arranged in an alphabetical order and are enumerated 
Table 1. Socio-demographic data of the tea tribe informants of Morigaon district, Assam $(\mathrm{N}=53)$

\begin{tabular}{|c|c|c|c|c|}
\hline SI. No. & Variable & Categories & No. of informants & Frequency (\%) \\
\hline \multirow{2}{*}{1.} & \multirow{2}{*}{ Gender } & Male & 39 & $73.58 \%$ \\
\hline & & Female & 14 & $26.41 \%$ \\
\hline \multirow{5}{*}{2.} & \multirow{5}{*}{ Age } & $20-30$ years & 6 & $11.32 \%$ \\
\hline & & 30 - 40 years & 9 & $16.98 \%$ \\
\hline & & $40-50$ years & 13 & $24.52 \%$ \\
\hline & & $50-60$ years & 18 & $33.96 \%$ \\
\hline & & $60-75$ years & 7 & $13.20 \%$ \\
\hline \multirow{4}{*}{3.} & \multirow{4}{*}{ Category of the informants } & Indigenous people & 53 & $100 \%$ \\
\hline & & Traditional health practitioners & 17 & $13.20 \%$ \\
\hline & & Laborer & 32 & $79.24 \%$ \\
\hline & & Service man & 4 & $7.54 \%$ \\
\hline \multirow{5}{*}{4.} & \multirow{5}{*}{$\begin{array}{l}\text { Experience of the Traditional } \\
\text { Health Practitioner }\end{array}$} & $\leq 1$ year & 1 & $1.89 \%$ \\
\hline & & $1-10$ years & 4 & $7.54 \%$ \\
\hline & & $10-20$ years & 7 & $13.20 \%$ \\
\hline & & 20 - 30 years & 3 & $5.66 \%$ \\
\hline & & $\geq 30$ years & 2 & $3.77 \%$ \\
\hline \multirow{2}{*}{5.} & \multirow{2}{*}{ Literacy rate } & Literate & 17 & $32.07 \%$ \\
\hline & & Illiterate & 36 & $67.92 \%$ \\
\hline
\end{tabular}

along with their correct botanical names, family, parts used, mode of preparation, and route of administration, UV and FL are summarized in Table 2. A total of 39 medicinal plants belonging to 36 genera and 27 families recorded to be used by the tea tribes as a remedy for jaundice in the study area.

The medicinal plant species documented from the study were distributed amongst 27 families and 36 genera. The highest numbers of plants were documented in the family Lamiaceae (5) followed by Leguminosae (3) and Acanthaceae (2), Oxalidaceae (2), Phyllanthaceae (2), Piperaceae (2), Poaceae (2) and Rutaceae (2) shown in Figure 2. Plant parts used by the tea tribes to treat jaundice were mainly aerial part, leaf, stem, bark of stem, fruit, rhizome, root, bark of root and whole plant. The most frequently used plant portion for the traditional remedies was the leaf (35.89\%) trailed by fruit $(17.94 \%)$, stem and whole plant (12.82\%), aerial part of plant, rhizome, root (5.12\%), calyx, flower, seed, stem bark and root bark (2.56\%) (Figure 3). There is no specific time to collect the plant materials used for herbal therapy as reported by the informants of the study area. Amongst the 39 medicinal plant species recognized, $33.33 \%$ are herbs followed by shrubs and trees $(25.64 \%)$ and lastly climbers and under-shrubs (7.69\%) (Figure 4).

Several forms of remedial preparation are used by the tea tribes in the study area and the most frequently used preparation was juice or extract with $43.58 \%$ trailed by infusions $(20.51 \%)$, paste $(15.38 \%)$, decoction and powder (7.69\%), concoction and garland (5.12\%) (Figure 5). The commonly used route of administration was oral $(94.87 \%)$ followed by external or topical use $(2.56 \%)$ and both oral and external use (2.56\%).

Most of the plants documented during the field study are wild in nature and are also available in the estates, residences, roadside, river-side and in hills and forest. Aloe vera, Averrhoa carambola, Cajanas cajan, Curcuma longa, Hibiscus sabdariffa, Lawsonia inermis, Mentha arvensis, Passiflora edulis, Piper nigrum, Saccharum officinarum are mainly cultivated 
Table 2. Ethno-medicines used against jaundice by tea tribes of Morigaon district, Assam

\begin{tabular}{|c|c|c|c|c|c|c|c|c|c|}
\hline $\begin{array}{l}\text { Sl. } \\
\text { No. }\end{array}$ & $\begin{array}{c}\text { Name of Plant } \\
\text { and Collection } \\
\text { Number }\end{array}$ & Family & Local name(s) & Habit & $\begin{array}{l}\text { Part(s) } \\
\text { used }\end{array}$ & $\begin{array}{l}\text { Mode of } \\
\text { prepa- } \\
\text { ration(s) }\end{array}$ & $\begin{array}{l}\text { Route } \\
\text { of } \\
\text { admint- } \\
\text { ration }\end{array}$ & $\begin{array}{l}\text { Use } \\
\text { Value } \\
\text { (UV) }\end{array}$ & $\begin{array}{l}\text { Fidelity } \\
\text { Level \% } \\
\text { (FL) }\end{array}$ \\
\hline 1. & $\begin{array}{c}\text { Achyranthes aspera } \\
\text { L. RBNG-03 }\end{array}$ & Amaranthaceae & $\begin{array}{c}\text { Ubhotakata } \\
\text { (Ass); Apamargo } \\
\text { (Ass and TT) }\end{array}$ & $\begin{array}{l}\text { Under- } \\
\text { shrub }\end{array}$ & Stem & $\begin{array}{l}\text { Worn as } \\
\text { garland } \\
\text { (chest } \\
\text { length } \\
\text { long). }\end{array}$ & External & 0.15 & 100 \\
\hline 2. & $\begin{array}{c}\text { Aegle marmelos (L.) } \\
\text { Correa RBNG-02 }\end{array}$ & Rutaceae & Bel (Ass and TT) & Tree & Leaf & Infusion & Oral & 0.15 & 87.5 \\
\hline 3. & $\begin{array}{c}\text { Aloe vera (L.) } \\
\text { Burm.f. RBNG-01 }\end{array}$ & Xanthorrhoeceae & $\begin{array}{c}\text { Salkuwori (Ass); } \\
\text { Ghritokumari } \\
\text { (Ass and TT); } \\
\text { Dahidahiya (TT) }\end{array}$ & Herb & Leaf & $\begin{array}{l}\text { Juice/ } \\
\text { Ex-tract }\end{array}$ & Oral & 0.09 & 60 \\
\hline 4. & $\begin{array}{l}\text { Alstonia scholaris } \\
\text { (L.) R.Br. RBNG-05 }\end{array}$ & Apocynaceae & $\begin{array}{c}\text { Satiana } \\
\text { (Ass and TT); } \\
\text { Rani Gamari (TT) }\end{array}$ & Tree & Stem & Powder & Oral & 0.15 & 75 \\
\hline 5. & $\begin{array}{l}\text { Argemone } \\
\text { mexicana L. } \\
\text { RBNG-25 }\end{array}$ & Papaveraceae & $\begin{array}{c}\text { Siyalkata } \\
\text { (Ass and TT); } \\
\text { Udishmari (TT) }\end{array}$ & Herb & Leaf & $\begin{array}{l}\text { Juice/ } \\
\text { Ex-tract }\end{array}$ & Oral & 0.05 & 33.3 \\
\hline 6. & $\begin{array}{l}\text { Averrhoa } \\
\text { carambola } \\
\text { L.RBNG-52 }\end{array}$ & Oxalidaceae & $\begin{array}{c}\text { Kordoi (Ass and } \\
\text { TT) }\end{array}$ & Tree & Fruit & $\begin{array}{l}\text { Juice/Ex- } \\
\text { tract }\end{array}$ & Oral & 0.09 & 57.1 \\
\hline 7. & $\begin{array}{l}\text { Azadirachta indica } \\
\text { A. Juss RBNG-20 }\end{array}$ & Meliaceae & $\begin{array}{c}\text { Mohaneem (Ass); } \\
\text { Neem (TT) }\end{array}$ & Tree & Leaf & Paste & Oral & 0.11 & 71.4 \\
\hline 8. & $\begin{array}{l}\text { Cajanus cajan (L.) } \\
\text { Millsp. RBNG-09 }\end{array}$ & Leguminosae & $\begin{array}{l}\text { Rahar (Ass); } \\
\text { Arhar (TT) }\end{array}$ & Shrub & Leaf & $\begin{array}{l}\text { Juice/Ex- } \\
\text { tract }\end{array}$ & Oral & 0.05 & 66.6 \\
\hline 9. & $\begin{array}{l}\text { Cassia fistula } \\
\text { L.RBNG-06 }\end{array}$ & Leguminosae & $\begin{array}{l}\text { Sonaru (Ass); } \\
\text { Sonari gocho } \\
\text { (TT) }\end{array}$ & Tree & Seed & Decoction & Oral & 0.05 & 66.6 \\
\hline 10. & $\begin{array}{c}\text { Cheilocostus } \\
\text { speciosus (J.Konig) } \\
\text { C. Specht RBNG-08 }\end{array}$ & Costaceae & $\begin{array}{c}\text { Jomlakhuti } \\
\text { (Ass); God (TT) }\end{array}$ & $\begin{array}{l}\text { Under- } \\
\text { shrub }\end{array}$ & $\begin{array}{l}\text { Rhi- } \\
\text { zome }\end{array}$ & Decoction & Oral & 0.07 & 100 \\
\hline 11. & $\begin{array}{c}\text { Clerodendrum } \\
\text { indicum (L.) Kuntze } \\
\text { RBNG-18 }\end{array}$ & Lamiaceae & $\begin{array}{l}\text { Akal bih (Ass); } \\
\text { Nagrikhari (TT) }\end{array}$ & Shrub & $\begin{array}{l}\text { Leaf, } \\
\text { Stem }\end{array}$ & $\begin{array}{l}\text { 1.Juice/ } \\
\text { Extract } \\
\text { (leaf) } \\
\text { 2. Worn as } \\
\text { garland } \\
\text { (stem) }\end{array}$ & $\begin{array}{l}\text { Oral and } \\
\text { external }\end{array}$ & 0.07 & 28.5 \\
\hline 12. & $\begin{array}{c}\text { Clerodendrum } \\
\text { infortunatum L. } \\
\text { RBNG-10 }\end{array}$ & Lamiaceae & $\begin{array}{c}\text { Bhet-tita (Ass); } \\
\text { Kumotia (TT) }\end{array}$ & $\begin{array}{l}\text { Under- } \\
\text { shrub }\end{array}$ & Leaf & Paste & Oral & 0.09 & 100 \\
\hline 13. & $\begin{array}{c}\text { Coffea } \\
\text { benghalensis B. } \\
\text { Heyne ex Schult. } \\
\text { RBNG-47 }\end{array}$ & Rubiaceae & $\begin{array}{c}\text { Sagoliphul (Ass } \\
\text { and TT) }\end{array}$ & Shrub & Root & Powder & Oral & 0.05 & 100 \\
\hline
\end{tabular}




\begin{tabular}{|c|c|c|c|c|c|c|c|c|c|}
\hline 14. & $\begin{array}{l}\text { Curcuma longa L. } \\
\text { RBNG-13 }\end{array}$ & Zingiberaceae & $\begin{array}{l}\text { Halodhi (Ass); Haldhi } \\
\text { (TT) }\end{array}$ & Herb & $\begin{array}{l}\text { Rhi- } \\
\text { zome }\end{array}$ & $\begin{array}{l}\text { Paste or } \\
\text { Powder }\end{array}$ & Oral & 0.09 & 80 \\
\hline 15. & $\begin{array}{c}\text { Cynodon } \\
\text { dactylon(L.) Pers. } \\
\text { RBNG-21 }\end{array}$ & Poaceae & $\begin{array}{l}\text { Dubori bon (Ass and } \\
\text { TT); Dub bon (TT) }\end{array}$ & Herb & $\begin{array}{l}\text { Whole } \\
\text { plant }\end{array}$ & Paste & Oral & 0.11 & 42.8 \\
\hline 16. & $\begin{array}{c}\text { Drymaria cordata } \\
\text { (L.) Willd. ex Schult. } \\
\text { RBNG-23 }\end{array}$ & $\begin{array}{c}\text { Caryo- } \\
\text { phyllaceae }\end{array}$ & Laijabori (Ass and TT) & Herb & $\begin{array}{l}\text { Whole } \\
\text { plant }\end{array}$ & Infusion & Oral & 0.22 & 90.9 \\
\hline 17. & $\begin{array}{l}\text { Ficus racemosa L. } \\
\text { RBNG-19 }\end{array}$ & Moraceae & $\begin{array}{c}\text { Dimoru (Ass and TT); } \\
\text { Dimri (TT) }\end{array}$ & Tree & Fruit & Decoction & Oral & 0.05 & 66.6 \\
\hline 18. & $\begin{array}{c}\text { Glycosmis } \\
\text { pentaphylla (Retz.) } \\
\text { DC. RBNG-16 }\end{array}$ & Rutaceae & $\begin{array}{c}\text { Chaul-dhowa, Bon } \\
\text { nemu (Ass); Bon } \\
\text { nimbu, Ashhoura (TT) }\end{array}$ & Shrub & Leaf & $\begin{array}{l}\text { Juice/ } \\
\text { Extract }\end{array}$ & Oral & 0.07 & 75 \\
\hline 19. & $\begin{array}{l}\text { Hibiscus sabdariffa } \\
\text { L. RBNG-26 }\end{array}$ & Malvaceae & $\begin{array}{c}\text { Khaseng-tenga, } \\
\text { Mesta-tenga (Ass); } \\
\text { Tengamora (Ass and } \\
\text { TT) }\end{array}$ & Shrub & $\begin{array}{l}\text { Calyx } \\
\text { and } \\
\text { Fruit }\end{array}$ & $\begin{array}{l}\text { Juice/ } \\
\text { Ex-tract }\end{array}$ & Oral & 0.07 & 75 \\
\hline 20. & $\begin{array}{l}\text { Houttuyunia cordata } \\
\text { Thunb. RBNG-29 }\end{array}$ & Saururaceae & $\begin{array}{c}\text { Masundori (Ass and } \\
\text { TT) }\end{array}$ & Herb & Leaf & Infusion & Oral & 0.07 & 60 \\
\hline 21. & $\begin{array}{c}\text { Hydrocotyle } \\
\text { sibthorpioides Lam. } \\
\text { RBNG-30 }\end{array}$ & Araliaceae & $\begin{array}{c}\text { Sarumani-muni (Ass); } \\
\text { Chhoto-manimuni } \\
\text { (TT) }\end{array}$ & Herb & $\begin{array}{l}\text { Whole } \\
\text { plant }\end{array}$ & $\begin{array}{l}\text { Juice/Ex- } \\
\text { tract }\end{array}$ & Oral & 0.09 & 55.5 \\
\hline 22. & $\begin{array}{l}\text { Justicia adhatoda L. } \\
\text { RBNG-04 }\end{array}$ & Acanthaceae & $\begin{array}{l}\text { Vahak (Ass); Vasak, } \\
\text { Basanti, Hej (TT) }\end{array}$ & Shrub & Leaf & $\begin{array}{l}\text { Juice/Ex- } \\
\text { tract }\end{array}$ & & 0.07 & 44.4 \\
\hline 23. & $\begin{array}{c}\text { Justicia gendarussa } \\
\text { Burm. f. RBNG-32 }\end{array}$ & Acanthaceae & $\begin{array}{c}\text { Jatrasiddhi (Ass); } \\
\text { Kaliadama, Brindaban } \\
\text { (TT) }\end{array}$ & Shrub & Leaf & Paste & Oral & 0.15 & 100 \\
\hline 24. & $\begin{array}{c}\text { Lawsonia inermis L. } \\
\text { RBNG-34 }\end{array}$ & Lythraceae & $\begin{array}{c}\text { Jetuka (Ass); Mehndi } \\
\text { (TT) }\end{array}$ & Shrub & Root & $\begin{array}{l}\text { Con- } \\
\text { coction }\end{array}$ & Oral & 0.07 & 100 \\
\hline 25. & $\begin{array}{l}\text { Leucas aspera } \\
\text { (Willd.) RBNG-31 }\end{array}$ & Lamiaceae & Durun (Ass and TT) & Herb & Leaf & $\begin{array}{l}\text { Juice/ } \\
\text { Extract }\end{array}$ & Oral & 0.05 & 60 \\
\hline 26. & $\begin{array}{c}\text { Mangifera indica L. } \\
\text { RBNG-33 }\end{array}$ & Anacardiaceae & Aam (Ass and TT) & Tree & $\begin{array}{l}\text { Bark of } \\
\text { Stem }\end{array}$ & Paste & Oral & 0.07 & 30.7 \\
\hline 27. & $\begin{array}{c}\text { Mentha arvensis L. } \\
\text { RBNG-40 }\end{array}$ & Lamiaceae & $\begin{array}{c}\text { Podina (Ass); Pudina } \\
\text { (TT) }\end{array}$ & Herb & $\begin{array}{c}\text { Aerial } \\
\text { part }\end{array}$ & $\begin{array}{l}\text { Juice/Ex- } \\
\text { tract }\end{array}$ & Oral & 0.05 & 50 \\
\hline 28. & $\begin{array}{c}\text { Ocimum tenuiflorum } \\
\text { L. RBNG-35 }\end{array}$ & Lamiaceae & $\begin{array}{c}\text { Koliyatulosi (Ass); Tulsi } \\
\text { (TT) }\end{array}$ & Shrub & Leaf & $\begin{array}{l}\text { Infusion } \\
\text { of crushed } \\
\text { leaves }\end{array}$ & Oral & 0.13 & 58.3 \\
\hline 29. & $\begin{array}{l}\text { Oxalis corniculata } \\
\text { L.RBNG-43 }\end{array}$ & Oxalidaceae & $\begin{array}{l}\text { Tengesi tenga (Ass); } \\
\text { Jhunjhuni (TT) }\end{array}$ & Herb & $\begin{array}{l}\text { Whole } \\
\text { Plant }\end{array}$ & $\begin{array}{l}\text { Juice/ } \\
\text { Extract }\end{array}$ & Oral & 0.07 & 44.4 \\
\hline 30. & $\begin{array}{l}\text { Passiflora edulis } \\
\text { Sims RBNG-41 }\end{array}$ & Passifloraceae & $\begin{array}{l}\text { Lota-bel (Ass); Kointho } \\
\text { (TT) }\end{array}$ & Climber & $\begin{array}{l}\text { Flower } \\
\text { and } \\
\text { Fruit }\end{array}$ & $\begin{array}{l}\text { Juice/ } \\
\text { Extract }\end{array}$ & Oral & 0.09 & 83.3 \\
\hline 31. & $\begin{array}{l}\text { Peperomia pellucida } \\
\text { (L.) Kunth RBNG-36 }\end{array}$ & Piperaceae & Pananua (Ass and TT) & Herb & $\begin{array}{c}\text { Aerial } \\
\text { part }\end{array}$ & $\begin{array}{l}\text { Juice/Ex- } \\
\text { tract }\end{array}$ & Oral & 0.02 & 33.3 \\
\hline 32. & $\begin{array}{c}\text { Phyllanthus emblica } \\
\text { L. RBNG-43 }\end{array}$ & Phyllanthaceae & $\begin{array}{c}\text { Amlokhi (Ass and TT); } \\
\text { Amlokhu (TT) }\end{array}$ & Tree & Fruit & $\begin{array}{l}\text { Juice/Ex- } \\
\text { tract }\end{array}$ & Oral & 0.09 & 45.4 \\
\hline
\end{tabular}




\begin{tabular}{|c|c|c|c|c|c|c|c|c|c|}
\hline 33. & $\begin{array}{l}\text { Phyllanthus } \\
\text { fraternus G.L. } \\
\text { Webster } \\
\text { RBNG-39 }\end{array}$ & Phyllanthaceae & $\begin{array}{c}\text { Mati-amlokhi (Ass); } \\
\text { Bhui-aaola (TT) }\end{array}$ & Herb & $\begin{array}{l}\text { Whole } \\
\text { Plant }\end{array}$ & Infusion & Oral & 0.11 & 85.7 \\
\hline 34. & $\begin{array}{l}\text { Piper nigrum L. } \\
\text { RBNG-43 }\end{array}$ & Piperaceae & $\begin{array}{c}\text { Jaluk (Ass); } \\
\text { Gul-morich (TT) }\end{array}$ & Climber & Fruit & Concoction & Oral & 0.07 & 44.4 \\
\hline 35. & $\begin{array}{l}\text { Plumbago } \\
\text { zeylanica L. } \\
\text { RBNG-46 }\end{array}$ & Plumbaginaceae & $\begin{array}{c}\text { Agyasit (Ass); } \\
\text { Agniboth, Chitamool, } \\
\text { Raiputi (TT) }\end{array}$ & Shrub & $\begin{array}{c}\text { Bark of } \\
\text { root }\end{array}$ & Infusion & Oral & 0.07 & 57.1 \\
\hline 36. & $\begin{array}{l}\text { Saccharum } \\
\text { officinarum L. } \\
\text { RBNG-48 }\end{array}$ & Poaceae & Kuhiyar (Ass and TT) & Herb & Stem & $\begin{array}{l}\text { Juice/ } \\
\text { Ex-tract }\end{array}$ & Oral & 0.09 & 100 \\
\hline 37. & $\begin{array}{l}\text { Tamarindus indica } \\
\text { L. RBNG-49 }\end{array}$ & Leguminosae & Teteli (Ass); Tetul (TT) & Tree & Fruit & Infusion & Oral & 0.04 & 66.6 \\
\hline 38. & $\begin{array}{l}\text { Tinospora sinensis } \\
\text { (Lour.) Merr. } \\
\text { RBNG-24 }\end{array}$ & Menispermaceae & $\begin{array}{c}\text { Amar-lota (Ass); } \\
\text { Guduchi, Choidoguni, } \\
\text { Amar-lot, Gulchhi (TT) }\end{array}$ & $\begin{array}{c}\text { Clim- } \\
\text { ber }\end{array}$ & Stem & Infusion & Oral & 0.13 & 77.7 \\
\hline 39. & $\begin{array}{l}\text { Xylosma longifolia } \\
\text { Clos. RBNG-51 }\end{array}$ & Salicaceae & $\begin{array}{c}\text { Mota-koli (Ass); } \\
\text { Kataponial } \\
\text { (Ass and TT) }\end{array}$ & Tree & Leaf & $\begin{array}{l}\text { Juice/ } \\
\text { Ex-tract }\end{array}$ & Oral & 0.17 & 69.2 \\
\hline
\end{tabular}

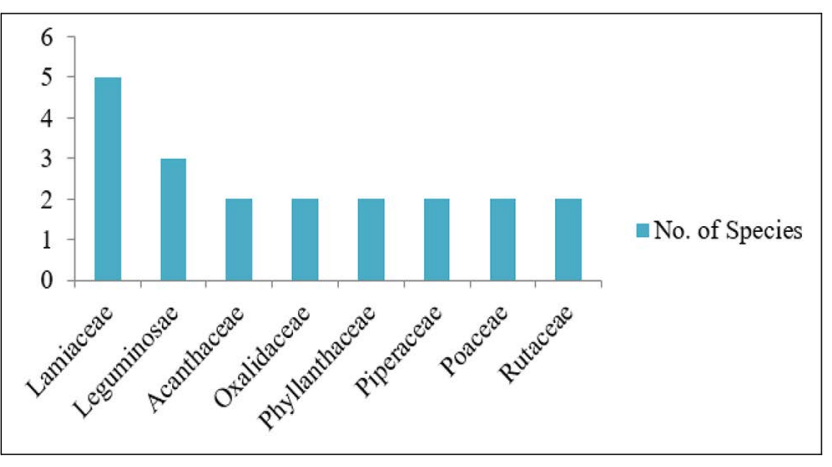

Figure 2. Dominant of the distributed medicinal plants.

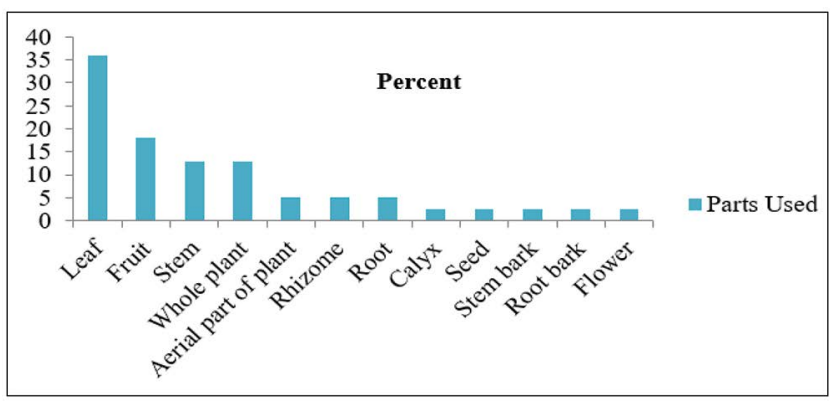

Figure 3. Plant parts used in the study area against jaundice.

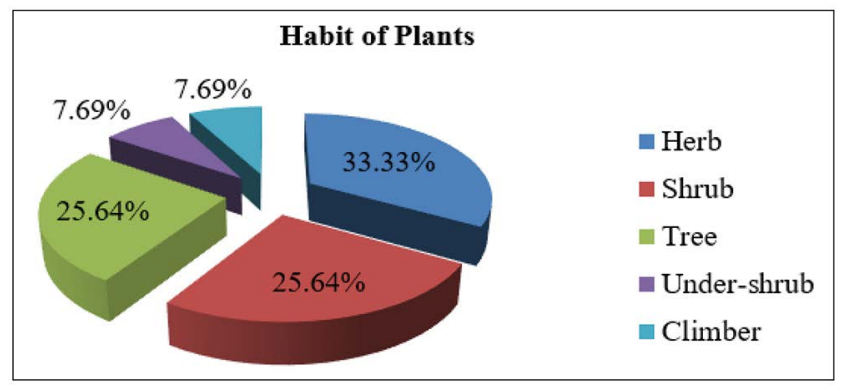

Figure 4. Habit of the plant species recorded in thestudy area.

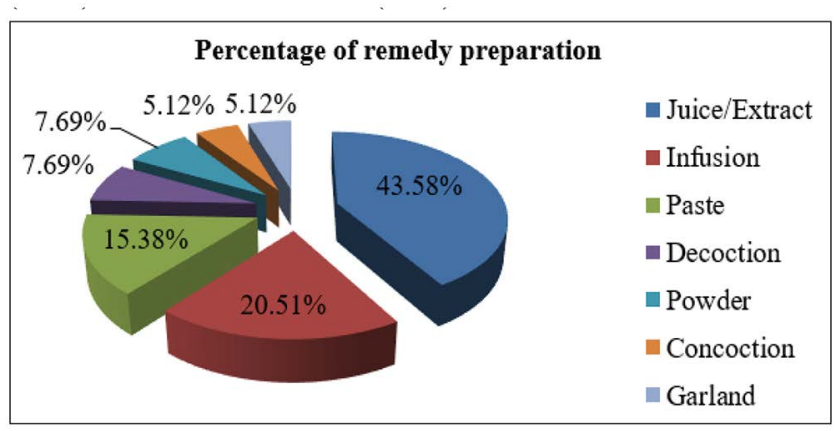

Figure 5. Mode of remedy preparation. 
in the study area. During the field study, systematic and proper care was taken in the collection methods.

This is the first quantitative ethnobotanical study conducted in this study area The highest UV ranked the Drymaria cordata (0.22) first, followed by Xylosma longifolia (0.17), Achyranthes aspera, Aegle marmelos, Alstonia scholaris and Justicia gendarussa (0.15) as second and third respectively. The Family Use Value (FUV) ranged between the lowest values (0.05) and the highest value (0.30). Lamiaceae family $(0.30)$ was the highest FUV trailed by Caryophyllaceae (0.22) and Rutaceae (0.18) shown in Table 3. The high values

Table 3. FUV of the plant families calculated from the actual and estimated UV of the plant species

\begin{tabular}{ll}
\hline Family & FUV \\
\hline Acanthaceae & 0.15 \\
Amaranthaceae & 0.15 \\
Anacardiaceae & 0.07 \\
Apocynaceae & 0.15 \\
Araliaceae & 0.09 \\
Caryophyllaceae & 0.22 \\
Costaceae & 0.07 \\
Lamiaceae & 0.30 \\
Leguminosae & 0.12 \\
Lythraceae & 0.07 \\
Malvaceae & 0.07 \\
Meliaceae & 0.11 \\
Menispermaceae & 0.13 \\
Moraceae & 0.05 \\
Oxalidaceae & 0.13 \\
Papaveraceae & 0.05 \\
Passifloraceae & 0.09 \\
Phyllanthaceae & 0.15 \\
Piperaceae & 0.05 \\
Plumbaginaceae & 0.07 \\
Poaceae & 0.16 \\
Rubiaceae & 0.05 \\
Rutaceae & 0.18 \\
Salicaceae & 0.16 \\
Saururaceae & 0.07 \\
Xanthorrhoeceae & 0.09 \\
Zingiberaceae & 0.09 \\
\hline
\end{tabular}

of UV and FUV can be explained by the fact that these plants are highly used and acknowledged by the majority of the informants in the study area. The Fidelity Level (FL) of the recorded plants have been calculated which showed the highest percentage of $100 \%$ for Achyranthes aspera, Cheilocostus speciosus, Clerodendrum infortunatum, Justicia gendarussa, Lawsonia inermis, Coffea benghalensis and Saccharum officinarum followed by Drymaria cordata (90.9\%) and Aegle marmelos (87.5\%).

In Morigaon district, jaundice is a common problem among the tea tribes due to their poor food habits, preventive care, and hygiene. They have gained the traditional knowledge on medicinal plants from the elders of their family and the village chiefs by word of mouth. However, there is a threat to the indigenous knowledge due to modernization; the knowledge may eventually be lost following the decease of the elderly and knowledgeable inhabitants of the study area. The documentation of this traditional knowledge is valuable for the indigenous people and their future generations for the wider use of the plants against jaundice. The majority of the informants were males $(73.58 \%)$ and females (26.41\%). Most of the ethno-pharmacological studies confirm that leaves are the major parts used for treating jaundice and resembles the present study $\underline{14}, \underline{28}, \underline{29}$. Most informants preferred to use fresh juice or extract of the plants to treat jaundice. The plants used by the informants are mostly wild in nature $(71.8 \%)$ while the cultivated plants represent only (28.2\%).

\subsection{A Brief Review on the Hepato- protective Activity of the Documented Plants of the Study}

In-vivo and in-vitro hepatoprotective activity of crude extracts of 29 of the 39 plant species identified in this study have been reported in the literature namely Achyranthes aspera, Aegle marmelos, Aloe vera, Alstonia scholaris, Argemone mexicana, Averrhoa carambola, Cajanus cajan, Cassia fistula, Cheilocostus speciosus, Clerodendrum infortunatum, Curcuma longa, Cynodon dactylon, Ficus racemosa, Glycosmis pentaphylla, Justicia adhatoda, Justicia gendarussa, Lawsonia inermis, Mentha arvensis, Passiflora edulis, Ocimun tenuiflorum, Oxalis corniculata, Phyllanthus emblica, Phyllanthus fraternus, Piper nigrum, Plumbago 
Table 4. Literature review of hepatoprotective effects of the documented plants of the study area

\begin{tabular}{|c|c|}
\hline Name of the species & Hepatoprotective activity \\
\hline Achyranthes aspera L. & $\begin{array}{l}\text { Effect of methanolic extract on rifampicin-induced hepatotoxicity and bi-herbal } \\
\text { ethanolic extract against paracetamol induced hepatic damage in albino rats } \underline{30}, \underline{31}\end{array}$ \\
\hline Aegle marmelos (L.) Correa & Effect of the methanolic extract of leaves in paracetamol intoxicated albino rats $\underline{32}$ \\
\hline Aloe vera (L.) Burm.f. & $\begin{array}{l}\text { Hepatoprotective activity of the gel against paracetamol induced hepatotoxicity in } \\
\text { albino rats } \underline{33}\end{array}$ \\
\hline Alstonia scholaris (L.) R.Br. & Effect of methanolic extract of stem bark $\underline{34}$ \\
\hline Argemone mexicana L. & In vivo activity of aerial part extract on $\mathrm{CCl}_{4}$ induced liver damage in wister rats $\underline{35}$ \\
\hline Averrhoa carambola L. & Effect against $\mathrm{CCl}_{4}$ and paracetamol induced liver toxicity $\underline{36}, \underline{37}$ \\
\hline Azadirachta indica A. Juss & Protective effect of leaf extract in rats against paracetamol induced hepatic damages $\underline{38}$ \\
\hline Cajanas cajan (L.) Huth & Effects on tissue defense system in D-galactosamine induced hepatitis in rats $\underline{39}, \underline{40}$ \\
\hline Cassia fistula L. & $\begin{array}{l}\text { Effect of aqueous extract of leaf and stem against } \mathrm{CCl}_{4} \text { induced liver damage in } \\
\text { rats } \underline{41}-\underline{43}\end{array}$ \\
\hline $\begin{array}{l}\text { Cheilocostus speciosus (J.Konig) C. } \\
\text { Specht }\end{array}$ & Hepatoprotective effect on paracetamol induced liver injury $\underline{44}$ \\
\hline Clerodendrum indicum (L.) Kuntze & No reference \\
\hline Clerodendrum infortunatum L. & Potentiality of methanol extract against $\mathrm{CCl}_{4}$ induced hepatotoxicity in rats $\underline{45}$ \\
\hline $\begin{array}{l}\text { Coffea benghalensis (Roxb. ex. } \\
\text { Schult.) J.-F.Leroy }\end{array}$ & No reference \\
\hline Curcuma longa $\mathrm{L}$. & $\begin{array}{l}\text { Protective effects on } \mathrm{CuSO}_{4} \text { induced hepatotoxicity and nephrotoxicity. } \\
\text { Immunomodulatory and hepatoprotective properties of aqueous extract against } \mathrm{CCl}_{4} \\
\text { intoxicity in swiss albino mice } \underline{46}, \underline{47}\end{array}$ \\
\hline Cynodon dactylon (L.) Pers. & Protective effects of aerial plants against $\mathrm{CCl}_{4}$ induced hepatotoxicity in rats $\underline{48}$ \\
\hline Drymaria cordata (L.)Willd.ex Schult. & No reference \\
\hline Ficus racemosa $\mathrm{L}$. & $\begin{array}{l}\text { Activity of leaf extract on liver damage in rats caused by } \mathrm{CCl}_{4} . \text { And hepatoprotective } \\
\text { effects of stem bark in albino rats against } \mathrm{CCl}_{4} \text { hepatic damages } \underline{49}, \underline{50}\end{array}$ \\
\hline Glycosmis pentaphylla (Retz.) DC. & Activity against paracetamol induced hepatotoxity in swiss albino rats $\underline{51}$ \\
\hline Hibiscus sabdariffa L. & No reference \\
\hline Houttuyunia cordata Thunb. & No reference \\
\hline Hydrocotyle sibthorpioides Lam. & No reference \\
\hline Justicia adhatoda L. & $\begin{array}{l}\text { Hepatotoprotective activity of aqueous, ethanol and methanol extracts against liver } \\
\text { injury in albino mice caused by } \mathrm{CCl}_{4} \underline{\underline{52}}\end{array}$ \\
\hline Justicia gendarussa Burm. f. & $\begin{array}{l}\text { In vitro hepatoprotective effects of stem against hepatotoxicity induced by } \mathrm{CCl}_{4} \text { in } \\
\text { rats } \underline{53}\end{array}$ \\
\hline Lawsonia inermis $\mathrm{L}$. & $\begin{array}{l}\text { Effect of warm aqueous extract in wister rats in } \mathrm{CCl}_{4} \text { and } 2 \text {-acetylaminoflourene } \\
\text { induced hepatic injury } \underline{54} \underline{55}\end{array}$ \\
\hline Leucas aspera (Willd.) & No reference \\
\hline Mangifera indica L. & No reference \\
\hline Mentha arvensis L. & Protective effect of leaves against $\mathrm{CCl}_{4}$ induced liver damages in rat $\underline{56}$ \\
\hline Ocimum tenuiflorum L. & Alcoholic effect of leaf extract against paracetamol induced liver damage in rats $\underline{57}$ \\
\hline Oxalis corniculata L. & $\begin{array}{l}\text { Activity of ethanolic extract against paracetamol induced hepatotoxicity in wister } \\
\text { rats } \underline{58}\end{array}$ \\
\hline Passiflora edulis Sims & Hepatoprotective effect of peel extract of the Passion fruit in albino rat $\underline{59}$ \\
\hline Peperomia pellucida (L.) Kunth & No reference \\
\hline Phyllanthus emblica L. & Hepatoprotective effects of ethanol extract $\frac{60}{}$ \\
\hline
\end{tabular}




\begin{tabular}{|c|c|}
\hline Phyllanthus fraternus G.L. Webster & $\begin{array}{l}\text { Effect of whole plant extracts on liver of Staphylococcus aureus intoxicated albino rats } \\
\underline{61}\end{array}$ \\
\hline Piper nigrum L. & Effect of methanolic extract against ethanol- $\mathrm{CCl}_{4}$ hepatotoxicity in wister rats $\underline{62}$ \\
\hline Plumbago zeylanica L. & Effect on paracetamol induced liver toxicity in rats $\underline{\underline{63}}$ \\
\hline Saccharum officinarum L. & Effect of juice in isoniazed induced hepatotoxicity in male albino rats $\underline{64}$ \\
\hline Tamarindus indica $\mathrm{L}$. & Ameliorating, anti-oxidant and hepatoprotective activity $\underline{65}, \underline{66}$ \\
\hline Tinospora sinensis (Lour.) Merr. & Aqueous extract hepatoprotective activity $\underline{\underline{67}}$ \\
\hline Xylosma longifolia Clos. & No reference \\
\hline
\end{tabular}

zeylanica, Saccharum officinarum, Tamarindus indica and Tinospora sinensis (Table 4).

Plant species like Clerodendrum indicum, Drymaria cordata, Hibiscus sabdariffa, Houttuyunia cordata, Hydrocotyle sibthorpioides, Leucas aspera, Mangifera indica, Peperomia pellucida, Coffea benghalensis and Xylosma longifolia are needed to analyze their hepatoprotective and other biological properties for development of some novel phyto-therapeutic active compounds.

\section{Conclusion}

The field studies carried out in the tea gardens of Morigaon district have led to the documentation of ethnomedicinal uses for 39 plant species. The reports provided by the tea tribal people of the study area, certainly increases the validity of the said purpose. From the above results and discussions, the tea tribes of the Morigaon district are still dependent on wild plants to cure jaundice. From the best of our knowledge Coffea benghalensis has not been reported earlier as a remedy for jaundice from Northeast India. The most widely used plants against jaundice recorded in this study such as Drymaria cordata, Xylosma longifolia, Achyranthes aspera, Cheilocostus speciosus, Clerodendrum infortunatum, Justicia gendarussa, Lawsonia inermis, Coffea benghalensis, Saccharum officinarum and Aegle marmelos and some plant species that have not yet been analyzed for the hepatoprotective properties should be prioritized for further phytochemical and pharmacological studies to identify the unknown compounds and their functional groups. Isolation of the bioactive compounds and elucidation of their chemical structures will help to develop new medicines for the treatment of jaundice in the future.

\section{Acknowledgements}

We are highly indebted to the tea tribe people of the selected study area for sharing their valuable indigenous knowledge. Prof. D. Borthakur from Department of Molecular Biosciences and Bioengineering, University of Hawai'i at Manoa is highly acknowledged for his invaluable support in the present work. We thank Dr. Smarajit Ojah from the Department of Geography of Nowgong Girls' College (Nagaon) for drawing the Morigaon district geographical map.

\section{Conflict of Interests}

No conflict of interest.

\section{References}

1. Grønhaug TE, Glæserud S, Skogsrud M, Ballo N, Bah S, Diallo D, Paulsen BS. Ethnopharmacological survey of six medicinal plants from Mali, West-Africa. Journal of Ethnobiology and Ethnomedicine. 2008; 4:26. https://doi.org/10.1186/17464269-4-26. PMid:19111068, PMCid:PMC2633328.

2. Sofowora A. Medicinal Plants and Traditional Medicine in Africa, John Wiley and Sons, New York, USA; 1982.

3. Lucy H, Edgar JD. Medicinal plants: A re-emerging health aid, division of life sciences UNESCO; 1999.

4. Tefera BN, Kim YD. Ethnobotanical study of medicinal plants in the Hawassa Zuria district, Sidama zone, Southern Ethiopia. Journal of Ethnobiology and Ethnomedicine. 2019; 15:25. https://doi.org/10.1186/s13002-019-0302-7. PMid:31 126296, PMCid:PMC6534827. 
5. Karunamoorthi K, Tsehaye E. Ethnomedicinal knowledge, belief and self-reported practice of local inhabitants on traditional antimalarial plants and phytotherapy. J. Ethnopharmacol. 2012; 141(1):143-150. https://doi.org/10.1016/j.jep.2012.02.012, https://doi.org/10.1016/j.jep.2012.02.022.

6. Balunas MJ, Kinghorn AD. Drug discovery from medicinal plants. Life Sci. 2005; 78:431-441. https://doi.org/10.1016/j. lfs.2005.09.012. PMid:16198377.

7. Maikhuri RK, Gangwar AK. Ethnobiological notes on the Khasi and Garo tribes of Meghalaya, Northeast India. Econ. Bot. 1993; 47:345. https://doi.org/10.1007/BF02907348.

8. Prakash JW, Anpin Raja RD, Asbin Anderson N, Williams C, Regini GS, Bensar K, Rajeev R, Kiruba S, Jeeva S, Das SSM. Ethnomedicinal plants used by Kani tribes of Agasthiyarmalai biosphere reserve, southern Western Ghats. Indian J. Traditional Knowledge. 2008; 7(3):410-413.

9. Timmermans K. Intellectual property right and traditional medicine: policy dilemmas at the interface. Soc. Sci. Med. 2003; 57(4):745-756. https://doi.org/10.1016/S0277-9536(02)004252 .

10. Dutta BK, Dutta PK. Potential of ethnobotanical studies in North East India: An overview. Indian J. Traditional Knowledge. 2005; 4(1):7-14.

11. Mao AA, Roy DK. Ethnobotanical studies in Northeast India: A review. In: Jain AK ed. Indian Ethnobotany: Emerging trends. Scientific publisher, India; 2016. p. 99-112.

12. Roche SP, Kobos R. Jaundice in the adult patient. Am. Fam. Physician. 2004; 69(2):299-304.

13. Abbas MW, Shamshad T, Ashraf MA, Javaid R. Jaundice: a basic review. Int. J. Res. Med. Sci. 2016; 4:1313-1319. https:// doi.org/10.18203/2320-6012.ijrms20161196.

14. Sharma J, Gairola S, Gaur RD, Painuli RM. The treatment of jaundice with medicinal plants in indigenous communities of the Sub-Himalayan region of Uttarakhand, India. J. Ethnopharmacol. 2012; 143:262-291. https://doi. org/10.1016/j.jep.2012.06.034. PMid:22759701.

15. Handa SS, Sharma A, Chakraborty K. Natural products as liver protecting drugs. Fitoterapia.1986; 57:307-351.

16. Narzary H, Brahma S, Basumatary S. Wild edible vegetables consumed by Bodo Tribe of Kokrajhar district (Assam), North-East India. Arch. Appl. Sci. Res. 2013; 5:182-190.

17. Das RK. Plantation Labour in India. Prabashi Press, India; 1931.

18. Griffiths P. The history of Indian tea industry. Weidenfield and Nicolson, London; 1967.

19. Martin GJ. Ethnobotany. A "people and plants" conservation manual. World Wide Fund for Nature, Chapman and Hall, London; 1995.

20. Jain SK. Methods and approaches in ethnobotany. Deep Publication, New Delhi; 1989.

21. Kanjilal UC, Das A, Kanjilal PC, De RN. Flora of Assam, Vol 1-5. Government of Assam, Shillong; 1934-1940.
22. Jain SK, Rao RR. A handbook of field and herbarium technique. Today and Tomorrow Publication, New Delhi; 1977.

23. The Plant List (The Plant List Version 1.1, 2013) Accessed in 1st April 2018. www.theplantlist.org.

24. Friedman J, Yaniv Z, Dafni A, Palewitch D. A preliminary classification of the healing potential of medicinal plants, based on the rational analysis of ethnopharmacological field survey amongBedouinsin NegevDesert, Israel.J.Ethnopharmacol.1986; 16:275-287. https://doi.org/10.1016/0378-8741(86)90094-2.

25. Alexiades MN, Sheldon JW. Selected guidelines for ethnobotanical research: A field manual. The New York Botanical garden, Boranx, New York. 1996; p. 99-133.

26. Tardio J, Pardo-de-Santayana M. Cultural importance indices: a comparative analysis bases on the useful wild plants of southern Cantabria (Northern Spain). Econ. Bot. 2008; 62:24-39. https://doi.org/10.1007/s12231-007-90045.

27. Zougagh S, Belghiti A, Rochd T et al. Medicinal and aromatic plants used in traditional treatment of the oral pathology: The ethnobotanical survey in the economic capital Casablance, Morocco (North Africa). Natural Products and Bioprospecting. 2019; 9:35. https://doi.org/10.1007/s13659018-0194-6. PMid:30484065, PMCid:PMC6328421.

28. Bhattacharyya R, Medhi KK, Borkataki S. Ethnomedicinal plants used against jaundice by the tea tribes of Nagaon district, Assam. Ethnobotany. 2016; 27:36-42.

29. Maruthupandian A, Mohan VR, Kottaimuthu R. Ethnomedicinal plants used for the treatment of diabetes and Jaundice by Palliyar tribals in Sirumalai hills, Western Ghats, Tamilnadu, India. Indian Journal of Natural Products and Resources. 2011; 2(4):493-497.

30. Bafna A, Mishra SH. Effect of methanol of Achyranthes aspera Linn. on rifampicin-induced hepatotoxicity in rats. Ars. Pharmaceutica. 2004; 45(4): 343-351 (2004).

31. Krishna Chaitanya DA, Reddy Challa S, Reddy AM. Hepatoprotective effect of biherbalethanolic extract against paracetamol induced hepatic damage in albino rats. J. Ayurveda Integr. Med. 2012; 3(4):198-203. https://doi.org/10.4103/09759476.104436. PMid:23326091, PMCid:PMC3545240.

32. Ramamurthy V, Raveendran S, KumarHVA, Hepatoprotective activity of the methanolic extract of Aegle marmelos leaves in paracetamol intoxicated albino rats. Int. J. Universal Pharmacy and Bio. Sci. 2014; 3(2):1-10.

33. Nayak V, Gincy TB, Prakash M, Joshi C, Rao S, Somayaji SN.Hepatoprotective activity of Aloe vera gel against paracetamol induced hepatotoxicity in albino rats. Asian J. Phar. Biol. Res. 2011; 1(2):94-98.

34. Kumar A, Khan MA, Saxena A, Singh RB. Hepatoprotective activity of methanolic extract of stem of Alstonia scholaris (L.)R. Br. Am. J. Pharma. Tech. Res. 2012; 2:2. 
35. Adam SIY, Idris IA, Abdelgadir EH, Ahmed RH, Kamal EE. Evaluation of hepatoprotective activity of Argemone mexicana aerial part extract on $\mathrm{CCl} 4$ induced liver damage in wister rats. British Journal of Pharmacology and Toxicology.2011; 2(5):251-256.

36. Ajeem AK, Mathew M, Nair C and Dilip C. Hepatoprotective effect of Averrhoa carambola fruit extract on carbon tetrachloride induced hepatotoxicity in mice. Asian Pacific Journal of Tropical Medicine. 2012; 3(8):610-613. https:// doi.org/10.1016/S1995-7645(10)60148-4.

37. Kumar GR, Prema P, Gupta P. Hepatoprotective activity of Averrhoa carambola against paracetamol induced liver toxicity in mice. International Journal of Botany Studies. 2016; 1(7):4-6.

38. Chattopadhyay R, Bandyopadhyay M. Possible mechanism of hepatoprotective activity of Azadirachta indica leaf extract against paracetamol induced hepatic damage in rats: Part III. Indian J. Pharmacol. 2005; 37:184-185. https://doi. org/10.4103/0253-7613.16218.

39. Akinloye OA, Olaniyi MO. Hepato-protective effect of Cajanus cajan on tissue defense system in D-galactosamineinduced hepatitis in rats. Turk J. Biochem. 2011; 36:237-241.

40. Gogoi JC, Mohanta D, Borah PK. Hepatoprotective Activity of Averrhoa carambola, Cajanus cajan and Paederia foetida against Acetaminophen and D-Galactosamine induced hepatotoxicity in rats. Journal of Pharmaceutical Research. 2010; 9(2):76-80. https://doi.org/10.18579/jpcrkc/2010/9/2/79491.

41. Hui Mei L, Hsien Chun T, Chau Jong W, Jin-Jin L, Chia Wen L, Fen-Pi C. Hepatoprotective effects of Cassia fistula Linn extract against $\mathrm{CCl} 4$ induced oxidative damage in rats. Chem. Biol. Interact. 2008; 171:283-293. https://doi. org/10.1016/j.cbi.2007.08.008. PMid:18045581.

42. Wasu SJ, Muley BP. Hepatotective effect of Cassia fistula. Ethnobotanical Leaflets. 2009; 13:910-916.

43. Chaudhari NB, Chittam KP, Patil VR. Hepatoprotective activity of Cassia fistula seeds against paracetamol-induced hepatic injury in rats. Arch. Pharm. Sci. Res. 2009; 1:218221.

44. AlSaadi BH, AlHarbi SH, Ibrahim SRM, El-Kholy AA, El-Agamy DS, Mohamed GA. Hepatoprotective activity of Costusspeciosus (koen. Ex. Retz.) against paracetamolinduced liver injury in mice. Afr. J. Tradit. Complement Altern. Med. 2018; 15(2):35-41. https://doi.org/10.21010/ ajtcamv15i2.5, https://doi.org/10.21010/ajtcam.v15i2.5.

45. SannigrahiS,MazumderUK,PalD,MishraSL.Hepatoprotective potential of methanol extract of Clerodendrum infortunatum Linn. against $\mathrm{CCl} 4$ induced hepatotoxicity in rats. Phcog. Mag. 2009; 5(20):394-399.

46. Sengupta M, Sharma GD, Chakraborty $B$. Hepatoprotective and immunomodulatory properties of aqueous extract of Curcuma longa in carbon tetrachloride intoxicated swiss albino mice. Asian Pac. J. Trop. Biomed. 2011; 1(3):193-199. https://doi.org/10.1016/S2221-1691(11)60026-9.

47. HashishEA, ElgamiSA. Hepatoprotectiveand nephroprotective effect of Curcumin against copper toxicity in rats. Indian J. Chin. Biochem. 2016; 31(3):270-277. https://doi.org/10.1007/ s12291-015-0527-8. PMid:27382197, PMCid:PMC4910845.

48. Surendra V, Prakash T, Sharma UR, Goli D, Dayalal S, Kotresha F. Hepatoprotective activity of aerial plants of Cynodon dactylon against CC14-induced hepatotoxicity in rats. J. Pharmacogn. Mag. 2008; 4:195-201.

49. MandalSC, MaityTK, Das J, Pal M, Saha BP. Hepatoprotective activity of Ficus racemosa leaf extract on liver damage caused by carbon tetrachloride in rats. Phytother. Res. 1999; 13(5):430-432. https://doi.org/10.1002/(SICI)10991573(199908/09)13:5<430::AID-PTR456>3.0.CO;2-G.

50. Ahmed F, Urooj A. Hepatoprotective effects of Ficus racemosa stem bark against carbon tetrachloride induced hepatic damage in albino rats. Pharmaceutical Biology. 2010; 48(2):210-216. https://doi.org/10.3109/13880200903081788. PMid: 20645843.

51. Nayak SS, Jain R, Sahoo AK, Hepatoprotective activity of Glycosmis pentaphylla against paracetamol-induced hepatotoxicity in swiss albino mice. Pharma Biol. 2011; 49(2):111-117. https://doi.org/10.3109/13880209.2010.501 084. PMid:20942640.

52. Afjal U, Gulfraz M, Hussain SS, Malix F. Hepatoprotective effects of Justicia adahtoda L. induced liver injury in Swiss albino mice. African Journal of Pharmacy and Pharmacology. 2013; 7:8-14. https://doi.org/10.5897/AJPP12.501.

53. Krishna KL, Mehta TA, Patel JA. In vitro hepatoprotective activity of Justicia gendarussa stem on isolated rat hepatocytes. Pharmacology Online. 2010; 2:9-13.

54. Hossain MC, Himangshu S, Maji, Chakraborty P. Hepatoprotective activity of Lawsonia inermis Linn. of warm aqueous extract in carbon tetrachloride induced hepatic injury in wister rats. Asian Journal of Pharmaceutical and Clinical Research.2011; 4:3.

55. Kumar M, Kaur P, Chandel M, Singh AP, Jain A, Kaur S. Antioxidant and hepatoprotective potential of Lawsonia inermis L. leaves against 2-acetylaminoflourene induced hepatic damage in male wister rats. BMC Complement Altern. Med. 2017; 17:56. https://doi.org/10.1186/s12906017-1567-9. PMid:28100199, PMCid:PMC5242058.

56. Patil K, Mall A. Hepatoprotective activity of Mentha arvensis Linn. leaves against $\mathrm{CCl} 4$ induced liver damage in rats. Asian Pacific Journal of Tropical Disease. 2012; 2(1):223226. https://doi.org/10.1016/S2222-1808(12)60156-X.

57. Lahon K, Das S.Hepatoprotective activity of Ocimum sanctum alcoholic leaf extract against paracetamolinduced liver damage in albino rats. Pharmacognosy Res. 2011; 3:13-18. https://doi.org/10.4103/0974-8490.79110. PMid:21731390, PMCid:PMC3119265. 
58. Sreejith G, Jayshree M, Latha PG, Suja SR, Shymal S, Shine VJ, Anuja G, Sini S, Shikha P, Krishnakumar NM, Vilash V, Shoumya S, Rajasekharan S. Hepatoprotective activity of Oxalis corniculata L. ethanolic extract against paracetamol induced hepatocity in wister rats and invite antioxidant effects. Indian J. Exp. Biol. 2014; 52(2):147-152.

59. Nerdy N, Ritarwan K. Hepatoprotective activity and Nephroprotective activity of peel extract from three varieties of the passion fruit (Passiflora sp.) in the albino rat. Open Access Maced. J. Med. Sci. 2019; 7(4):536-542. https://doi.org/10.3889/oamjms.2019.153. PMid:30894908, PMCid:PMC6420947.

60. Pramyothin P, Samosorn P, Poungshompoo S, Chaichantipyuth C. The protective effects of Phyllanthus emblica Linn. extract on ethanol induced rat hepatic injury. J. Ethnopharmacol. 2006; 107:361-364. https://doi.org/10 .1016/j.jep.2006.03.035. PMid:16750340.

61. Ramamurthy V, Abarna T. Hepatoprotective Activity of Phyllanthus niruri whole plant extracts against Staphylococcus aureus intoxicated Albino Rats. Global J. Biol Agricul. Health Sci. 2014; 3(3):256-260.

62. Nirwane AM, Bapat AR. Effect of methanolic extract of Piper nigrum fruits in ethanol-CCl4 induced hepatotoxicity in wistar rats. Der. Pharmacia Lettre. 2012; 4:795-802.
63. Kanchana N, Sadiq AM. Hepatoprotective effect of Plumbago zeylanica on paracetamol induced liver toxicity in rats. Int. J. Pharm Sci. 2011; 3:32-39.

64. Khan SW, Tahir M, Lone KP, Munir B, Latif W. Protective effect of Saccharum officinarum L. (sugarcane) juice on isonised induced hepatotoxicity in male albino mice. J. Ayub. Med. Coll. Abbottabad. 2015; 27(2):346-350.

65. Amir M, Khan MA, Ahmed S, Akhter M, Mujeeb M, Ahmed A. Ameliorating effects of Tamarindus indica fruits extract on anti-tubercular drugs induced liver toxicity in rats. Nat. Prod. Res. 2015; 30(6):715-719. https://doi.org/1 0.1080/14786419.2015.1039001. PMid: 25978515.

66. Rodriguez Amado JR, Lafourcade-Prada A, Escalona-Arranz JC, Peraz-Roses R, Morris-Quevedo H, Keita H. Antioxidant and hepatoprotective activity of a new tablet formulation from Tamarindus indica L. Evid. Based Complement Alternat. Med. 2016; 7. https://doi.org/10.1155/2016/3918219. PMid:27143986, PMCid:PMC4838804.

67. Kumar V, Modi VK, Saxena KK. Exploration of hepatoprotective activity of aqueous extract of Tinospora cordifolia- an experimental study. Asian J. Pharm. Clin. Res. 2013; 6(1):87-91. 